\title{
Sensory contrast effects in the judgment of lifted weights'
}

MAUREEN Y. WILLIAMS, JOHN ROSS AND VINCENT DI LOLLO

THE UNIVERSITY OF WESTERN AUSTRALIA

Contrast effects based on changes in the sensory characteristics of lifted weights were demonstrated. Two groups of 10 Ss were trained to select three reference weights. Ss then lifted either a heavy "shift" series (Group H) or a light "shift" series of weights (Group L), and were then required to reproduce the original reference weights. After the shift Group H selected heavier weights and Group L lighter weights than in the pre-shift settings.

Stevens (1958) has suggested that contrast effects in sensory experiments involving categorical judgments reflect changes in the reference system under which judgments are made, and not changes in the apparent magnitude (AM) of the stimuli. The confounding between effects on AM and on the reference system may be described as follows. If only two response categories are available ("heavy" and "light"), the reference system can be characterized by one boundary (B) between the two categories. A given weight will thus be judged "heavy" if AM > B and "light" if AM $<$ B. The boundary $\left(B_{1}\right)$ set by $S s$ judging a light series of weights may differ from that set by Ss judging a heavier series $\left(B_{h}\right)$, so that $B_{1}<B_{h}$. Following a shift from one series of weights to another, the category boundary may change in level but may fall short of the level set by a control group judging the second series throughout. Consequently, differences in the proportion of "heavy" judgments made by shifted and control groups may occur due to differences in the level of category boundaries, regardless of changes in AM. Judgmental contrast effects obtained following shifts in the heaviness of lifted weights (Di Lollo, 1964) may therefore have confounded the effects of changes in level of category boundaries and changes in sensory characteristics.

In the present study Ss were first trained to locate three reference weights to a strictcriterion of learning. Each $S$ first chose a neutral (neither-heavy-nor-light) weight, and then two others by the method of "magnitude production." Training continued until $S$ could consistently produce each reference weight. They were then given experience with either a light or a heavy "shift" series of weights, and were finally required to reproduce (i.e., find again) the three reference weights under the instruction to produce the three weights originally chosen. They were not asked to choose the weights which currently met the original subjective criteria.

Since the Ss were required to reproduce well-known weights, both before and after the "shift" series, the difficulties of changing category boundaries are avoided. A difference between pre- and post-shift production of the reference weights indicates a sensory change in AM.
Method

Subjects. The Ss were 20 male students attending the introductory Psychology course at the University of Western Australia.

Apparatus. The weight-lifting apparatus was similar to that described by Von Wright \& Mikkonen (1964). It consisted of a cylindrical vertical aluminum rod 2-1/2 in. high and of 1 in. diameter which acted as the variable weight to be judged. The rod was attached at one end of a horizontal metal bar which was pivoted and anchored at the other end. Weight at the rod was varied by sliding a lead runner along the bar and could be altered by $\mathrm{E}$ or $\mathrm{S}$ turning a handle. The apparatus was contained in a wooden box $36 \times 12 \times 7$ in. high with only the rod protruding from the top and the two handles from the far sides. Weight in grams was indicated on a dial which could not be seen by $S$. The Ss were required to rest their forearm on the box and lift the weight with a motion of the wrist only.

Procedure. To familiarize the $\mathrm{Ss}$ with the range of weights, 20 weights (from $50 \mathrm{gm}$ to $1,000 \mathrm{gm}$ in steps of $50 \mathrm{gm})$ were presented three times in random order. To maintain attention to the weights, Ss were required to judge the second weight in each pair as heavier or lighter than the first. The Ss were then required to produce a weight which felt neither heavy nor light and to call it "100." Weights which felt half as heavy and twice as heavy were then selected and called 50 and 200 respectively. Each of these three "reference weights" was the mean of four judgments, two of which were made from heavier and two from lighter starting positions. Each $\mathrm{S}$ was then asked to learn to reproduce, from random starting positions, each of the three originally chosen reference weights to a criterion of four consecutive correct reproductions $( \pm 10 \%$ of the weight concerned). After meeting the criterion the Ss were allocated to two groups of $10 \mathrm{Ss}$ each. Group L was presented with a light shift series, and Group $\mathrm{H}$ with a heavy shift series. The shift series consisted of five weights, $25 \mathrm{gm}$ apart, centered around the 50 reference weight (Group L) or the 200 reference weight (Group $\mathrm{H}$ ). The five weights in the shift series were presented 10 times in random order, $\mathrm{S}$ being required to judge whether the second of two weights was heavier or lighter than the first. The Ss were then asked to reproduce each reference weight four times: twice from a heavier and twice from a lighter starting position. Each reference weight reported below is thus the mean of four settings averaged across the $10 \mathrm{Ss}$ in a group.

\section{Results and Discussion}

The post-shift reference settings for the heavy shift group are higher than the original setting and those for 


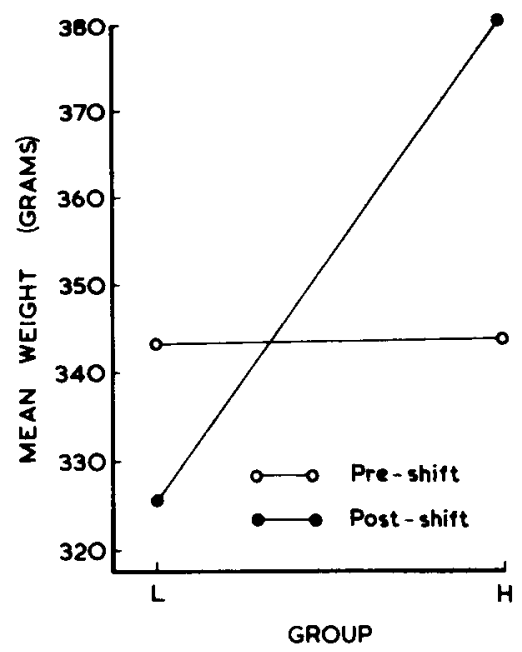

Fig. 1. Mean pre- and post-shift judgment for each group averaged over the three reference positions.

the light shift group lower. Figure 1 shows the weights set pre- and post-shift by both groups, averaged across the three reference levels; and Fig. 2 the weights set for each reference level separately. A 2 by 3 by 2 analysis of variance-groups by reference levels by (pre-and post-) shift (Winer, 1962, p. 320)-shows significant interactions for groups by shift $(F=14.63 ; \mathrm{df}=1 / 18 ; \mathrm{p}<0.01)$ and for reference level by shift $(F=5.63$; $d f=2 / 36 ; p<$ 0.01 ). The only significant main effect was for reference level.

The fact that, after the shift, the groups differ in weight settings when asked to reproduce the original reference levels supports Helson's (1964, p. 136) contention that a "profound change in sensory character" occurs when Ss are shifted from one series of weights to another. Before the shift Ss were able to reproduce their original settings reliably with no guide other than AM; after the shift the groups differed in their settings in the expected direction, which implies contrasting shifts in AM. Reference system effects are not involved by virtue of the design.

This experiment does not allow a determination of the relative amount of shift produced by the heavy and light series because it lacked control groups with no shift series or with a series centered around the 100 refer-

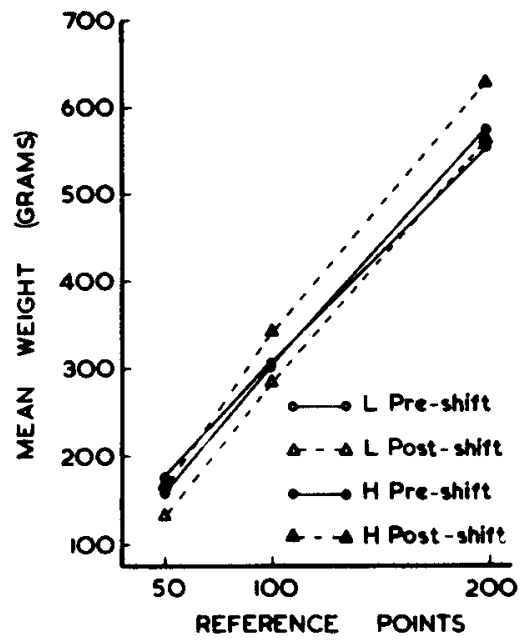

Fig. 2. Mean pre- and post-shift judgment for each group for each reference position.

ence level. However, the difference between the groups post-shift is sufficient to show a change in AM in at least one case, if not both. The technique used in this study can be readily extended to estimate the magnitude of AM shift effects in weight judgment and in the judgment of other stimulus characteristics.

\section{References}

Di Lollo, v. Contrast effects in the judgment of lifted weights. J. exp. Psychol., 1964, 68, 383-387.

Helson, H. Adaptation level theory. New York: Harper \& Row, 1964. Stevens, S. S. Adaptation-level vs. the relativity of judgment. Amer. J. Psychol., 1958, 71, 633-646.

Von Wright, J. M., \& Mikkonen, V. Changes in repeated reproduction of weight as a function of adaptation level. Scand. J. Psychol., $1964,5,239-248$.

Winer, B. J. Statistical principles in experimental design. New York: McGraw-Hill, 1962.

\section{Note}

1. This report is based on a dissertation submitted by the first author in partial fulfillment of the requirements for the degree of B. Psych. (Hons.) at the University of Western Australia. The work was done under the direction of the second and third authors. This research was sponsored by the Air Force Office of Scientific Research, Office of Aerospace Research, United States Air Force, under AFOSR Grant AF-AFOSR-968-65 to the third author. 